\title{
Les Cahiers d'Outre-Mer
}

Revue de géographie de Bordeaux

\section{Logiques d'acteurs et échelles de risques dans l'exploitation forestière au Cameroun}

\section{Roger Ngoufo et Maurice Tsalefac}

\section{(2) OpenEdition}

\section{Journals}

\section{Édition électronique}

URL : http://journals.openedition.org/com/206

DOI : $10.4000 /$ com.206

ISSN : 1961-8603

\section{Éditeur}

Presses universitaires de Bordeaux

\section{Édition imprimée}

Date de publication : 1 janvier 2006

Pagination : 115-132

ISBN : 978-2-86781-406-8

ISSN : 0373-5834

\section{Référence électronique}

Roger Ngoufo et Maurice Tsalefac, « Logiques d'acteurs et échelles de risques dans l'exploitation forestière au Cameroun », Les Cahiers d'Outre-Mer [En ligne], 233 | Janvier-Mars 2006, mis en ligne le 01 janvier 2009, consulté le 01 mai 2019. URL : http://journals.openedition.org/com/206 ; DOI : $10.4000 /$ com.206

Ce document a été généré automatiquement le 1 mai 2019.

(c) Tous droits réservés 


\title{
Logiques d'acteurs et échelles de risques dans l'exploitation forestière au Cameroun
}

\author{
Roger Ngoufo et Maurice Tsalefac
}

1 Les forêts du bassin du Congo en général et celles du Cameroun en particulier sont soumises à d'intenses pressions liées au développement de l'exploitation forestière. Il en résulte, d'après les « discours consacrés », une déforestation et une dégradation biologique de ces forêts. Or, le Cameroun doit préserver ses ressources forestières pour les générations futures, participer à la lutte contre les changements climatiques et concrétiser ses engagements internationaux sur la protection de l'environnement et de la biodiversité. En même temps, il doit aussi compter sur l'exploitation forestière pour réduire la pauvreté, aggravée depuis le début des années 1990. C'est dire que face au risque écologique global déclaré qui est la disparition des forêts et pour lequel la déforestation peut être considérée comme menace et aléa, s'ajoute le risque socio-économique imposé par la mondialisation et les exigences de bonne gouvernance des bailleurs de fonds internationaux et multilatéraux. Au niveau local, et face à la faiblesse de l'Etat se sont mis en place des réseaux sociaux (exploitants forestiers, Associations, ONG et société civile) qui imposent leur propre loi, déterminant une exploitation forestière minière. À partir des enquêtes de terrain et de l'exploitation des documents d'archives et cartographiques, il est montré qu'on se retrouve en présence d'un jeu d'intérêts sans merci, source de risques divers. Comment arriver à dégager les composantes du (des) risque( $s$ ) dans l'exploitation forestière au Cameroun? À cette question, nous essayons de répondre : en insistant sur les particularités et les enjeux que représentent les forêts camerounaises pour les différents acteurs ainsi que de la progression extraordinaire de son exploitation au cours de ces dernières années ; ensuite en identifiant les principaux exploitants de cet écosystème, leurs motivations et les risques qu'ils redoutent. Enfin, il est montré que le « risque » envisagé dans un contexte d'illégalité ne peut conduire qu'à l'incertitude totale et au « à qui mieux mieux » qui accélère la dégradation des ressources. 
Situation, contexte socio-économique et enjeux de l'exploitation forestièreSituation et enjeux de l'exploitation forestière

2 Le Cameroun, pays d'Afrique centrale situé au Nord de l'Équateur, sur le rebord occidental de la cuvette congolaise, s'étend du $2^{\circ}$ au $13^{\circ}$ de latitude Nord et du $9^{\circ}$ au $16^{\circ}$ de longitude Est (fig. 1). Ses forêts situées essentiellement au sud de $6^{\circ} \mathrm{N}$ comprennent 7 types : des forêts ombrophiles sempervirentes à longes feuilles en plaine (56\%), des forêts d'altitude $(1 \%)$, des forêts marécageuses $(1 \%)$, des mangroves $(1 \%)$, des forêts dites dégradées $(22 \%)$, des arbres épars $(8 \%)$ et des forêts ombrophiles décidues et semi décidues à larges feuilles (11\%). L'ensemble de ces forêts occupait une superficie d'environ 19,6 millions ha au milieu des années 1990.

3 Sur le plan de la biodiversité, les forêts camerounaises abritent plus de 8000 espèces de plantes supérieures et plus de la moitié des espèces d'oiseaux et de mammifères africains. Les forêts montagnardes abritent des espèces endémiques. Les forêts littorales sont particulièrement riches en plantes alors que les forêts de l'intérieur sont caractérisées par une grande diversité de mammifères.

4 Les forêts camerounaises contribuent également au maintien de la bonne qualité des eaux, et assurent le stockage du carbone (1,3 à 6,6 gigatonnes selon les évaluations) : le Cameroun, en menant une politique attentive à la production des forêts primaires résiduelles, rendrait au monde entier un service écologique de première importance puisqu'il est classé parmi les 15 pays tropicaux qui jouent un rôle important pour le captage et le stockage du carbone.

5 Sa production du bois de feu est considérable et essentielle pour la majeure partie de la population qui ne compte que sur elle pour son approvisionnement en énergie. Le bois de feu et le charbon de bois représentent à peu près $80 \%$ de la consommation totale d'énergie du pays.

6 La forêt camerounaise fournit également de la nourriture, des médicaments et des matériaux de construction à une grande partie de la population. Les produits forestiers non ligneux (mangue sauvage, écorce et fruits de Garcinia cola, noix de palme, noix de cola et poire africaine) et le gibier sont une source importante de l'alimentation des populations et de revenus pour les femmes pauvres. La crise économique du début des années 1990 a augmenté le prix des médicaments et accru la dépendance du monde rural vis-à-vis des plantes médicinales. La plupart de ces plantes n'existent qu'à l'état sauvage et sont soumises à de fortes pressions face à l'exploitation et à la conversion des forêts qui menacent leur habitat. Les routes forestières facilitent l'accès à des zones jusque-là excentrées, facilitant la chasse et les feux de forêt.

7 On estime que plus de 18 millions d'hectares de forêts originelles ont été défrichées à des fins agricoles et d'habitation. La déforestation aurait progressé au taux annuel de $0,6 \%$ an en moyenne sous l'action de l'agriculture et de l'exploitation forestière qui ouvrent de vastes massifs de forêts primaires (forêts - frontières) jusque là intactes. Ces forêts primaires représentaient $10 \%$ de la superficie forestière actuelle.

Contexte socio-économique de l'exploitation forestière

8 Le Cameroun, aujourd'hui l'un des pays pauvres très endettés d'Afrique, a connu depuis son indépendance trois grandes périodes d'évolution économique. De 1960 à 1979, sa croissance économique réelle impulsée par le secteur agricole, s'est en effet maintenue entre 3 et $5 \%$. À partir de 1979, l'avènement du pétrole a apporté un souffle nouveau à l'ensemble de l'économie et particulièrement à la production agricole. La croissance 
réelle de l'économie s'est accélérée pour atteindre des taux moyens de $7 \%$ environ jusqu'en 1985/86. Il s'en est suivi un accroissement des dépenses publiques, qui ont doublé entre 1980-1981 et 1986-1987.

9 À partir de 1986-1987, une grave crise économique a affecté les conditions de vie des populations. Jusqu'en 1993/94 les taux de croissance de l'ordre de -3 à - 4 \% ont obligé le gouvernement à recourir à des politiques budgétaires restrictives, induisant des réductions drastiques des dépenses en faveur des secteurs sociaux. Les compressions des personnels liées à la fermeture ou à la restructuration des entreprises des secteurs public, parapublic et privé ainsi que l'arrêt des recrutements dans la fonction publique ont accru le nombre de chômeurs.

10 Sous le double effet de la dévaluation du Franc CFA intervenue en janvier 1994, le Cameroun a renoué avec la croissance économique et le taux de croissance réelle est remonté entre 3 et $5 \%$ entre 1995-1996 et 1997-1998. Mais la reconstitution des revenus de la population et l'augmentation de la consommation se sont effectuées dans un contexte d'inflation qui a réduit les impacts positifs de cette reprise économique sur le niveau de vie réel des populations. D'où l'aggravation du chômage, l'accès difficile aux services sociaux essentiels (santé, éducation, eau potable, la dégradation des infrastructures économiques et sociales ainsi que de l'environnement en général) augmentant les risques d'exclusion des groupes sociaux vulnérables. En 1996, plus de la moitié de la population totale consommaient moins des 2400 calories par jour prescrits par la FAO comme étant le seuil minimum. À Douala et à Yaoundé, les revenus par tête ont baissé de l'ordre de 31 et $52 \%$ respectivement, alors qu'en milieu rural ceux des paysans, déjà structurellement bas, s'écroulaient à cause de la défaillance des mécanismes mis en place dans le but de soutenir les prix d'achat aux producteurs à l'occasion de la chute des cours mondiaux. L'austérité budgétaire et la privatisation entreprises par les pouvoirs publics n'ont donné lieu ni à une amélioration des revenus des populations ni à un secteur privé apte à prendre la relève en terme d'investissements, de création d'emplois et de richesses. Bien au contraire, elles se sont accompagnées du développement de la corruption qui a largement profité aux catégories sociales les plus proches du pouvoir en place.

11 Avant 1994, les ressources forestières étaient régies par le code forestier de 1981 et le décret d'application de 1983. Mais cette législation n'est pas parvenue à fournir un cadre juridique pour planifier l'aménagement des terres, l'intégration de la production forestière et sa conservation. Face à l'ambiance économique délétère la Banque Mondiale s'est efforcée d'améliorer la gestion des forêts dans la région en utilisant le Cameroun comme modèle pour élaborer un processus de réforme exigeant en contrepartie des prêts d'ajustement structurels. Cette réforme était axée sur des objectifs économiques, sociaux et écologiques contradictoires. Le 20 janvier 1994, le parlement a adopté une loi réglementant les activités forestières. Cette loi a été suivie du décret $\mathrm{N}^{\circ}$ 95/531/PM qui précise les modalités d'application afférentes à la foresterie et qui instaure 4 changements majeurs :

12 - l'attribution des concessions (type de droit d'exploitation) par un système d'enchères ; de nouveaux mécanismes de prix et de taxation;

13 - l'obligation d'avoir des plans d'aménagement ;

14 - et des dispositions en matière de foresterie communautaire. 
15 Entre autres dispositions, l'actuel code forestier prévoit en matière d'exploitation forestière que toute activité doit être soumise à l'approbation des autorités chargées des forêts et que les résidents au Cameroun ou les compagnies qui sont enregistrées dans ce pays, soient seuls éligibles.

16 L'exploitation forestière à l'intérieur du domaine Forestier Permanent se fait, dans les forêts domaniales, par le biais de ventes de coupes (vente d'un volume sur pied, un type de droit d'exploitation) ou de conventions d'exploitation. Cette dernière formule est habituellement désignée par le terme de "concession» et peut comporter une ou plusieurs Unités forestières d'Aménagement (UFA). Il s'agit d'unité de gestion forestière, sous-division d'un contrat d'exploitation. Comme établi lors de la réforme forestière en 1994, l'exploitation forestière à l'intérieur du domaine Forestier Non permanent s'effectue par des ventes de coupes, un permis ou une autorisation de récupération (un type de droit d'exploitation, attribué par volume).

Une exploitation forestière minière des forêts camerounaises

17 L'exploitation forestière a progressé rapidement depuis 1959 débordant le cadre des régions littorales et les alentours des grands centres urbains où elle était concentrée pour s'étendre dans le Sud et l'Est du pays. Moins d'un cinquième de la forêt non protégée reste à l'extérieur du front de l'exploitation forestière. Seul moins de $20 \%$ de la forêt non protégée du pays n'ont pas été exploités. Des concessions d'exploitation encerclent des aires protégées telle la Réserve du Dja, un site du patrimoine mondial. Certaines concessions se sont retrouvées dans un proche passé, à l'intérieur même des limites géographiques d'une aire protégée; c'est le cas de l'ancienne réserve de Campo qu'exploitait la Compagnie dite "Forestière de Campo », qui a vu ses limites redéfinies pour sa transformation en Parc National de Campo-Ma'an en 2000.

18 L'exploitation forestière ne se traduit pas toujours par des enlèvements d'arbres, mais bien plus par l'ouverture des zones auparavant inaccessibles à la colonisation agricole et à la chasse. Les forêts de la Cross River Korup, de la région Yaoundé - Ebolowa - Kribi ou de Bertoua - Abong - Mbang - Djoum qui incluent une partie importante de la forêt résiduelle du Cameroun sont désormais reconnues comme des zones à risques (hotspots) de déforestation.

19 Cette exploitation forestière camerounaise, essentiellement sélective, place le Cameroun parmi les 5 principaux exportateurs mondiaux de grumes tropicales et de deuxième exportateur de grumes tropicales du bassin du Congo derrière le Gabon. Le Cameroun a exporté 1,7 million de mètres cubes de grumes tropicales soit $10 \%$ du total mondial. Les exportations de bois industriel ont généré en moyenne 230 millions de dollars/an entre 1996 et 1998. Entre 1997 et 1998 le total des exportations a chuté de près d'un demimillion de mètres cubes.

20 Les pays européens ne sont plus les principaux importateurs : $51 \%$ des exportations sont destinées aux marchés asiatiques. Les principales essences exportées sont l'Ayous (30\%) le Sappeli $(7 \%) ; 80$ essences sur les 300 que l'on trouve dans le pays sont exploitées commercialement.

21 Les superficies exploitées ne sont cependant pas rapportées dans leur totalité du fait d'activités d'exploitation illicite ou d'archives officielles incomplètes. Les compagnies forestières extraient un volume de bois plus élevé par hectare pour satisfaire des marchés qui demandent une gamme plus large d'essences.

Les Acteurs de l'exploitation forestière au Cameroun 
22 Des individus aux multinationales, les acteurs de l'exploitation forestière sont nombreux et divers. Nous nous limiterons ici aux plus représentatifs en fonction de l'influence qu'ils exercent dans la gestion forestière au Cameroun: il s'agit des bailleurs de fonds internationaux et multilatéraux (Banque Mondiale, FMI) de l'Etat, des compagnies forestières, des populations locales des communes, des organismes d'appui (ONG et Projets), des élites et des institutions décentralisées.

Les bailleurs de fonds internationaux

23 Parce qu'ils ont prêté de l'argent à l'État en crise, ils estiment avoit un droit de regard sur la gestion des affaires nationales et notamment forestières. C'est ainsi que, dans le cadre de l'Ajustement structurel, s'est mise en place la Country Assistance Strategy composante forêt (CAS), instrument permettant à la Banque Mondiale d'évaluer le niveau de mise en application des reformes convenues lors de ses missions successives ou des échanges dette-nature à l'exemple de l'initiative PPTE (Pays Pauvres Très endettés) qui se matérialise à l'heure actuelle par l'obligation pour le pays élu de rédiger un document de Stratégie de Réduction de la Pauvreté (DSRP) dans un système de plus en plus libéralisé. Alors que, les bailleurs de fonds imposent des évaluations régulières et des conditions à l'État, ce dernier, partagé entre l'obligation d'honorer ses engagements et d'assurer ses fonctions régaliennes, dénonce l'iniquité des rapports Nord-Sud considérés comme principale cause de sa vulnérabilité. Surtout que, face à une population pauvre et dans un contexte de corruption se multiplient des sociétés prête-noms derrière lesquelles se cachent des étrangers pour maximiser les gains obtenus de l'exploitation forestière.

L'État

24 Il a engagé dès 1988 dans le cadre du Premier Programme d'Ajustement Structurel une série d'actions visant à contenir les incidences sociales de la crise économique. Ses actions se sont développées au début, principalement dans le cadre du projet Dimensions Sociales de l'Ajustement (DSA) et par la mise en place des réformes législatives et réglementaires orientées vers la libéralisation des activités économiques et le développement des initiatives privées génératrices de revenus. Toutefois la persistance de la récession économique a limité la portée et l'impact des actions engagées. Les pertes d'emploi et la baisse des revenus ont persisté posant à l'État des problèmes de trésorerie. C'est pourquoi dans le domaine de l'environnement, il a adopté un programme dit de Développement Humain Durable prenant explicitement en compte la dimension de l'environnement dans le cadre du Programme National de Gestion de l'Environnement (PNGE). L'objectif de ce programme est de développer des politiques, des stratégies et des actions pour la protection de l'environnement et la gestion rationnelle des ressources en vue de contribuer à un développement durable. L'État camerounais a aussi développé un système de fiscalité des ressources forestières pour parer à la baisse des revenus issus de l'exploitation du pétrole. La fiscalité forestière approvisionne le budget de l'État et contribue aux efforts d'ajustement du pays. Parmi les objectifs généraux de la politique forestière camerounaise, l'augmentation des recettes de l'État, des collectivités et des communautés locales figure en bonne place. Les éléments de cette fiscalité sont, de l'amont à l'aval de la filière :

25 - les redevances à la surface concédée ou exploitée- les redevances sur le volume, des taxes à l'exportation des grumes, des taxes à l'exportation des produits transformés. La taxe d'abattage est égale à $2,5 \%$ de la valeur FOB des grumes avec décote de $15 \%$ tandis que les droits de sortie des grumes sont égaux à $17,5 \%$ de cette même valeur soit 7 fois 
plus. Les sciages sont taxés à $12,5 \%$. Les droits de sortie des grumes représentent les $2 / 3$ de la fiscalité totale.

Pour un meilleur suivi de ces taxes, il faut disposer d'un service forestier fort motivé, formé, capable de faire respecter la réglementation en vigueur, de contrôler la mise en place des aménagements et plans de gestion. Cela pose le problème des moyens et de leur pérennité dans la durée. Or les capacités logistiques actuelles du Ministère de l'Environnement et des forêts sont très insuffisantes. Le personnel de terrain ne dispose pas de moyens de transport et de locaux et ne peut donc pas inspecter comme il se doit les concessions.

\begin{tabular}{|c|c|c|c|c|c|}
\hline Provinces & Est & Sud & Centre & Littoral & Sud-ouest \\
\hline Nombre d'agents & 116 & 115 & 232 & 167 & 163 \\
\hline $\begin{array}{c}\text { Nombre de } \\
\text { véhicule 4*4 }\end{array}$ & 1 & 1 & 1 & 1 & 1 \\
\hline Nombre de motos & 4 & 4 & 10 & 4 & 6 \\
\hline
\end{tabular}

L'État camerounais est donc tiraillé entre le besoin de préserver ses ressources forestières (conformément aux résolutions des grands sommets mondiaux sur l'environnement et le développement auxquels il a adhéré) et de contrôler leur exploitation (sans les moyens adéquats) et de jouer son rôle régalien qu'il ne peut pas assumer n'ayant aucune ressource. Il se doit également de répondre aux exigences des bailleurs de fonds. Écartelé entre plusieurs voies, il est obligé d'ignorer certains manquements des acteurs de l'exploitation forestière car trop de transparence dans ce domaine créerait des problèmes étant donné l'implication de certains hauts dignitaires proches du pouvoir. Il s'ensuit un certain laxisme, un certain laisser-faire pour ne pas troubler "l'ordre public», d'où le développement de la corruption à différents niveaux et des réseaux de relations intéressées qui font dire par les acteurs dans ce domaine que : « on est quelqu'un par son quelqu'un ». Ainsi les investissements attendus des exploitants forestiers en faveur des populations sont souvent détournés par ces réseaux, alors que tout autour d'eux règnent une misère et une pauvreté extrêmes (rares sont ceux qui gagnent 1 dollar/jour). L'État doit ainsi à la fois, envisager le maintien des grands équilibres macro-économiques, redouter les risques sociaux de la part d'une population pratiquement abandonnée à ellemême et susceptible de se révolter à tout moment, et être attentif aux conditions du service de la dette envers ses bailleurs de fonds tout en dénonçant l'iniquité des rapports Nord-Sud comme le facteur principal de sa vulnérabilité.

Les compagnies forestières

En 1998, 479 compagnies forestières étaient enregistrées contre 177 en 1990 et 106 en 1980. Cette évolution traduit une tendance à des investissements accrus dans l'industrie forestière. En 1999, 84 particuliers et compagnies avaient des permis d'exploitation en règle (licences, concessions ou ventes de coupe) à l'intérieur des forêts camerounaises. En pratique, il est difficile d'avoir une idée précise de l'identité des principaux acteurs de l'exploitation forestière pour deux raisons: la pratique généralisée de l'affermage de plusieurs filiales et le manque de renseignements au sujet des compagnies et des particuliers enregistrés comme étant titulaires des concessions. L'Observatoire Mondial des Forêts (OMF) a établi que près des $2 / 3$ de la superficies des concessions sont contrôlés en tout ou partie par des compagnies qui ne sont pas camerounaises et on ignore 
l'affiliation de $19 \%$ de titulaires de concessions enregistrés. Partiellement, parce qu'elles disposent de davantage de capitaux, les compagnies étrangères jouent un rôle économique disproportionné dans le secteur forestier. Selon le CIFOR (1996) les compagnies étrangères et les entreprises exportent plus de $70 \% \mathrm{du}$ bois d'œuvre et possèdent plus de la moitié des scieries et autres installations de transformation primaire, ayant les capacités les plus importantes. Les forêts camerounaises sont contrôlées par un petit groupe d'opérateurs qui, par leurs stratégies de gestion et leurs méthodes d'exploitation, pourraient affecter de manière significative l'avenir de cette ressource naturelle. En 1998-1999, trois compagnies forestières françaises (Thanry, Bolloré et Coron) détenaient près $\mathrm{du} 1 / 3$ de la superficie des concessions forestières camerounaises. Dix sociétés-mères, sont partiellement ou totalement financées par des intérêts français, et détiennent la moitié des concessions forestières du Cameroun (Tabl.2), soit plus de 100000 ha de forêt.

Le rapport de force s'est progressivement inversé au profit des sociétés asiatiques (Tableau 3). Ainsi, le groupe chinois Vicwood contrôle une superficie de près de 800000 ha soit 15,8 \% de la surperficie totale concédée en 2002-2003 (tabl ; III).

\begin{tabular}{|c|c|c|}
\hline Compagnie (filiales) & $\begin{array}{c}\text { Superficie des } \\
\text { concessions 1998-99 } \\
\text { (milliers ha) }\end{array}$ & $\begin{array}{l}\% \text { superficie totale des } \\
\text { concessions 1998-99 }\end{array}$ \\
\hline $\begin{array}{c}\text { Thanry (CIBC, SAB, SEBC, } \\
\text { CFC, Prenant) }\end{array}$ & 650 & 16 \\
\hline $\begin{array}{c}\text { Bolloré (La forestière de } \\
\text { Campo, SIBAF) }\end{array}$ & 412 & 10 \\
\hline Coron & 212 & 5 \\
\hline Alpi (Alpicam, Grumcam) & 204 & 5 \\
\hline Hazim (SFH) & 157 & 4 \\
\hline Rougier (SFID) & 132 & 3 \\
\hline Delcovenaere (SOTREF, SFIL) & 75 & 2 \\
\hline Itallegno (ECAM) & 69 & 2 \\
\hline Vasto Legneault (SEFAC) & 63 & 2 \\
\hline Pasquet (Pallisco) & 61 & 1 \\
\hline Autres & 2019 & 50 \\
\hline Total & 4050 & 100 \\
\hline
\end{tabular}




\begin{tabular}{|c|c|c|c|}
\hline $\begin{array}{l}\text { Groupes (Concession- } \\
\text { naires) }\end{array}$ & $\begin{array}{l}\text { Nationalité des } \\
\text { concessionnaires }\end{array}$ & Superficie (ha) & $\begin{array}{l}\text { \% de total } \\
\text { par super- } \\
\text { ficie }\end{array}$ \\
\hline $\begin{array}{l}\text { VICWOOD (CABC, CFC, } \\
\text { SEBC, Propalm, SAB, } \\
\text { SEBC, J.Prenant, Kief- } \\
\text { fer) }\end{array}$ & Chine & 787811 & 15,8 \\
\hline $\begin{array}{l}\text { ROUGIER (Lorema, } \\
\text { SFID,SOCIB, Cambois) }\end{array}$ & France & 474164 & 9,5 \\
\hline $\begin{array}{c}\text { SEFAC (La Filière } \\
\text { bois, SEBAC) }\end{array}$ & Italie & 411872 & 8,3 \\
\hline $\begin{array}{l}\text { KHOURY (EFMK, } \\
\text { SABM,SN COCAM) }\end{array}$ & Liban / Cameroun & 338093 & 6,8 \\
\hline $\begin{array}{c}\text { Bolloré } \\
\text { (SIBAF, HFC) }\end{array}$ & France & 314748 & 6,3 \\
\hline $\begin{array}{l}\text { ALPI (ALPICAM, } \\
\text { STBK, GRUMCAM) }\end{array}$ & Italie & 305019 & 6,1 \\
\hline $\begin{array}{c}\text { PASQUET } \\
\text { (Pallisco, Assene } \\
\text { Nkou, Sodetrancam) }\end{array}$ & France / Cameroun & 301387 & 6,0 \\
\hline $\begin{array}{c}\text { PATRICE BOIS (COFA, } \\
\text { SFF) }\end{array}$ & Italie / France & 206866 & 4,2 \\
\hline $\begin{array}{c}\text { WIJMA (COFA/WIJ- } \\
\text { MA) }\end{array}$ & Pays Bas / Cameroun & 203333 & 4,1 \\
\hline Autres** & & 1643089 & 33,0 \\
\hline TOTAL & & 4986380 & 100,0 \\
\hline
\end{tabular}

Tableau III- Les plus grands groupes (concessionnaires), par superficie en 2002-2003
Source : Interactive forestry atlas of Cameroon ; notes de Tauteur

Source: Interactive forestry atlas of Cameroon ; notes de l' ratteur
$* *$ toutes les societés forestieres quit ont moins de 200000 ha sous exploitation

Conme on le voit, L'État se trouve devant un dilemme : la nécessité de la sauvegarde de la biodiversité et l'impératif de financer le développement du pays? Faut-il minimiser les risques environnementaux par rapport aux risques socio-économiques? Ceci est une question particulièrement cruciale dans le contexte du développement durable, où l'on doit faire face à d'urgents problèmes de réduction de la pauvreté avec des ressources limitées.

Populations locales

problèmes même si au demeurant et très souvent les agriculteurs sont aussi des chasseurs.

Les agriculteurs

Bien que les forêts aient toujours été le principal domaine de leurs activités, elles ont redécouvert à la faveur de la crise économique l'écosystème forestier comme source de devises. Elles développent actuellement une agriculture intensive essentiellement tournée vers l'approvisionnement des villes et donc vers la recherche du gain. Des jeunes de retour des villes s'investissent dans l'agriculture. Ils utilisent des tronçonneuses pour créer des bananeraies commerciales modifiant ainsi l'économie du travail : il devient possible d'abattre la forêt avec un effort moindre. On est donc passé d'un type d'agriculture qu'on pouvait considérer comme écologiquement durable à un autre très prédateur de l'espace et des arbres. Certes, jusqu'à la crise économique du début des années 1990, le caféier et le cacaoyer avaient été intégrés à l'agriculture des populations forestières et étaient cultivés soit derrière les cases soit sous la forêt. On pouvait considérer qu'ils ne représentaient pas une menace pour la forêt, ces cultures servant de marqueurs de la propriété de la terre. Le paysage qui résultait de ce type d'agriculture était des noyaux "anthropogènes" dans une matrice de forêt peu influencée par l'homme et contenait une biomasse élevée en petits et moyens mammifères même avec 
une forte pression de chasse. La Chasse de subsistance, d'ailleurs adaptée à une telle situation, était bénéfique. En effet, la faune composée de rongeurs (aulacode, athérure, rat de Gambie) mais également de singes (cercocèbe) peut être considérée comme issue d'un élevage extensif dans la mesure où elle évolue dans un milieu aménagé par l'homme. Les chasseurs La crise économique a déterminé un taux de chasse au fusil bien supérieur à celui de la pratique de pièges traditionnels et concentré l'effort de chasse dans la forêt, loin des villages. Il s'agit d'activités de chasse commerciales qui concernent en premier lieu les mammifères de grande taille (céphalophe) dont la viande boucanée apporte des revenus réguliers: il en résulte qu'on constate presque partout l'extinction des grands mammifères à proximité des villages. Il importe donc de trouver des modèles de gestion de la forêt prenant en compte les aspirations des populations locales, motivant la conservation de la biodiversité et anticipant l'impact futur de l'accroissement de la population.

Face à l'exploitation forestière industrielle, les populations locales pensent, par rapport à la loi de 1994 réglementant l'exploitation forestière et qui leur accorde le bénéfice de $10 \%$ des redevances de superficie acquittées dans les UFA du domaine permanent, qu'elles doivent en recevoir plus pour le développement de leur communauté, d'autant qu'elles estiment avoir été exclues jusque-là du partage des bénéfices pécuniaires de la forêt. En outre sur les ventes de coupe, le paiement aux villageois d'une somme forfaitaire par mètre cube abattu a été introduit temporairement par l'administration dans le cahier de charges des exploitants. Les populations préfèrent passer au-dessus de la réglementation en vigueur et solliciter directement ce qui leur revient de l'exploitant, ce qui épargne à ce dernier les tracasseries administratives et le paiement de multiples pourboires auprès des autorités communales chargées de percevoir les taxes. Si pour les 2, les stratégies sont différentes, l'enjeu commun est économique et conjoncturel : bénéfice fort/risque faible en l'absence de contrôle. Cette connivence inédite entre les populations et les exploitants justifiée par l'appétit du gain dénote un manque de confiance vis-à-vis des autorités communales.

Avec les populations locales, la déforestation, la dégradation des ressources, le déclin et l'extinction de certaines espèces sont craints. Cependant, les interdictions d'extraction, de récolte ou de commerce peuvent aussi poser des risques à plus long terme ou des risques indirects, par exemple en terme de mécontentement des utilisateurs des ressources locales, de réduction ou d'élimination des incitations économiques à protéger les espèces ou de déclin de la volonté politique de protéger l'environnement. D'un autre côté les stratégies d'exploitation, qui ne posent pas de risques d'extinction immédiats d'espèces ciblées, peuvent présenter des risques à long terme de modification génétique ou de perturbation du fonctionnement des écosystèmes. Faut-il donc prendre en compte à la fois les risques pour la biodiversité à court terme (ou directs) et à long terme (ou indirects) ? Comment ceux-ci peuvent-ils être évalués et mis en balance?

Communes, Organismes d'appui (ONG et Projets), Élites, Institutions décentralisées :

bataille pour les parts du gâteau

De leur côté les élites adoptent deux attitudes opposées (Ngoufo, 2000) : d'un côté, elles s'appuient sur les ONG et associations pour exercer sur les exploitants forestiers des pressions diverses allant de l'exigence de la construction d'infrastructures locales sociales pour leur communauté à la demande de boycott au niveau international des produits et bois en provenance du Cameroun. De l'autre, elles font de la politique leur fonds de 
commerce et cherchent à s'approprier les Mairies pour gérer les taxes issues de l'exploitation forestière. Pour les premiers, le risque se décline en terme de crainte d'accaparement des terres par les étrangers; les seconds voudraient garder une forte emprise sur les populations et utiliser les bénéfices tirés de l'exploitation forestière à des fins électoralistes voire personnelles.

Discussion

Le concept de risque dans l'exploitation forestière au Cameroun recouvre des aspects si divers qu'il est difficile de les envisager tous. L'incertitude n'est pas simplement écologique (changement climatique, modification de la biodiversité), mais est également relative aux impacts potentiels de la mondialisation, de la décentralisation, des effets des mouvements des marchés mondiaux et des régimes de commerce.

Les connaissances scientifiques peuvent fournir une compréhension des facteurs biologiques et écologiques qui influencent le risque de surexploitation auquel fait par exemple face l'écosystème d'une espèce ou d'une forêt. Cependant, les espèces et les ressources sont exploitées par des humains qui prennent des décisions et procèdent à des allocations de ressources dans des contextes sociaux et économiques spécifiques. La logique des intérêts prime alors et la notion de risque devient très complexe dès lors qu'elle implique non seulement des groupes sociaux mais aussi des individus - dont les logiques ne sont pas toujours très évidentes - qui constituent en eux-mêmes un risque.

Dans ces conditions, certains auteurs (Cooney, 2003) ont préconisé la gestion adaptative (outil de gestion) pour gérer l'incertitude. R. Cooney met l'accent sur les prises de décision provisoires et révocables, avec un contrôle soigneux des impacts menant à une prise de décision perfectionnée. Quelle est la relation entre le principe de précaution (réponse à l'incertitude par des actions visant à éviter des dommages graves ou irréversibles) et la gestion adaptative ? Certains considèrent ces deux approches comme concurrentes, la précaution favorisant le fait de "ne rien faire" jusqu'à ce qu'il soit prouvé qu'un acte est sans risque et la gestion adaptative favorisant des interventions contrôlées qui augmentent la compréhension du système en question. Cependant, d'autres considèrent la gestion adaptative comme une stratégie intrinsèquement de précaution, étant donné qu'elle reconnaît que, dans les systèmes complexes, la certitude de l'issue est impossible et cherche à créer un régime dynamique capable de réagir à des changements imprévisibles. Les partisans de cette démarche considèrent la gestion adaptative comme un moyen essentiel pour mettre la précaution en œuvre aux niveaux national et local dans le domaine de la protection de l'environnement. La gestion adaptative est-elle une stratégie de précaution valable?

$\mathrm{Au}$ lendemain de la crise économique du début des années 1990 et de la dévaluation du franc CFA, le Cameroun, en butte à de sérieux problèmes de trésorerie s'est replié sur l'exploitation forestière pour répondre en partie aux conditions imposées par les bailleurs de fonds internationaux. Parallèlement, il a été obligé de se plier aux exigences de bonne gouvernance et de démocratisation prescrites par ces bailleurs de fonds. Cette situation a profondément affaibli l'État, principal sinon unique gestionnaire jusque-là des ressources forestières, et a favorisé la montée en puissance de multiples réseaux sociaux (Associations, ONG, élites) qui revendiquent non seulement un droit de regard mais aussi leur part dans la gestion des ressources forestières. Cette multiplicité des réseaux d'intérêt s'est nécessairement accompagnée de nouvelles formes de violences entre les différents protagonistes, sources de risques multiformes dont la compréhension passe par la multiplication des études monographiques. 


\section{BIBLIOGRAPHIE}

BIGOMBE LOGO P. et DABIRE ATAMANA B., 2003 - Gérer autrement les conflits forestiers au Cameroun. Yaoundé : Presses de l'UCAC, $214 \mathrm{p}$.

BIKIE H., COLLOMB J. G, DJOMO L, MINMEYER S., NGOUFO R et NGUIFFO S., 2000 - An overview of logging in Cameroon. Washington : Global Forest Watch, World Resources Institute, 66 p., 9 cartes couleurs. (http://www.globalforestwatch.org/common/cameroon/english/report.pdf/)

BIKIE H., COLLOMB J. G, DJOMO L, MINMEYER S., NGOUFO R et NGUIFFO S., 2005 - Atlas forestier interactif du Cameroun, version 1.0. Document de synthèse. Un rapport de Global Forest Watch 2005. Washington : World Resources Institute, 49 p. (http://www.globalforestwatch.org/french/ pdf/Document_synthese_francais. pdf/)

COLLOMB J.-G. et BIKIE H., 2001 - 1999-2000 Allocation of logging permits in Cameroon : finetuning Central Africa's first auction system. Washington : Global Torest Watch, 39

p. (www.globalforestwatch.org/common/french/allocation.pdf)

COONEY R, 2000 - Le principe de précaution et la gouvernance environnementale :

développement durable, gestion des ressources naturelles et conservation de la biodiversité. note internet projet UICN, $5 \mathrm{p}$.

MINEFI, Yaoundé, 2000 - Audit économique et financier du secteur forestier au Cameroun. Rapport provisoire CIRAD - Forêt/institutions et développement, inédit.

NGOUFO R. et TSALEFAC M., 2001 - Atomisation de l'espace et gestion du patrimoine forestier au Cameroun, du pouvoir colonial à l'Etat moderne. In : COSAERT P. et BART F., eds. - Patrimoines et développement dans les pays tropicaux. IXes Journées de Géographie Tropicale, La Rochelle, 13 et 14 septembre 2001. Pessac : Presses Universitaires de Bordeaux - DyMSET, p. 215-226 (Espaces Tropicaux, 18)

NGOUFO R., 2000 - Contraintes pratiques de mise en œuvre de la réglementation sur l'exploitation forestière au Cameroun. Rapport CEW/CARPE. Yaoundé : CEW/CARPE 97 p.

Revue Canopée, $n^{\circ} 18$, octobre 2000 et $n^{\circ} 24$, juillet 2003.

\section{RÉSUMÉS}

Les forêts du Bassin du Congo sont, après celles de l'Amazonie, les plus vastes forêts du monde. Renommées pour leur biodiversité, elles abritent des populations qui les exploitent à des fins diverses : alimentation, construction, santé. Au début des années 1990, le Cameroun qui possède certaines des forêts les plus diverses et les plus menacées, a pris l'initiative d'un processus de réforme de sa politique forestière en liaison avec un prêt d'ajustement structurel accompagné de la libéralisation, de la privatisation et de la violence du marché. À l'échelle internationale, le risque déclaré est celui de la disparition des forêts tropicales sous l'effet des processus de déforestation qui constituent la menace principale et en même temps l'aléa parce qu'il est le fait d'une conjonction de facteurs difficiles à maîtriser. Dans la pratique, l'iniquité des rapports NordSud fournit les principaux facteurs de vulnérabilité. L'État affaibli doit paradoxalement continuer à jouer son rôle régalien dans un contexte où les logiques de profit se trouvent exacerbés. Il 
redoute davantage une baisse des revenus fiscaux du secteur de nature à mettre en péril son fonctionnement interne et ses engagements externes vis-à-vis des bailleurs de fonds. Le secteur forestier au Cameroun offre donc la possibilité d'apprécier les imbrications du global et du local et inversement pour une lecture nouvelle des risques prenant en compte les motivations des acteurs.

The Congo basin forests are after that of the Amazonian, the largest forest of the world. They are well known for their biodiversity and dwelling populations that exploit them for diverse goals : Food, construction, health. At the beginning of the 1990, Cameroon took an initiative of forest policy reforms in accordance with a structural adjustment loan. This has been accompanied by the liberalization, privatization and market violence. At the international scale, the declared risk is that of the disappearance of tropical forest under the effect of deforestation processes that constitute a principal threat and at the same time a hazard because it is the combination of elements that are difficult to master. In practice, the North/South iniquity offers causes of risk as well as factors of vulnerability. A weakened State has to paradoxically continue to play its regulatory role in a context where the individual profits margins and interests are found exaggerated. A fiscal revenue reduction in the sector would disturb the internal functioning of the State and jeopardize its external engagements vis-avis donors. The forestry sector in Cameroon offers thus the possibility to appreciate global and local implications in a new approach to study risks taking into account the motivations of involved actors.

INDEX

Mots-clés : ajustement structurel, Cameroun, échelle de risques, exploitation forestière, logique d'acteurs

Keywords : actors logic, Cameroon, forest exploitation, scale of risk, structural adjustment 